\title{
COMUNICAÇÃO
}

\section{CONTROLE QUÍMICO DA "ERGOT" (Claviceps africana Frederickson, Mantle \& de Milliano) OU DOENÇA-AÇUCARADA E DAS PRINCIPAIS DOENÇAS FOLIARES DO SORGO (Sorghum bicolor (L.) Moench)}

\author{
NICÉSIO FILADELFO JANSSEN DE ALMEIDA PINTO ${ }^{1}$
}

\begin{abstract}
RESUMO - O controle químico da "ergot" (Claviceps africana) foi realizado em campo de produção de sementes do híbrido de sorgo granífero BR 304 (Sorghum bicolor (L.) Moench.), utilizando-se os fungicidas ( $\mathrm{g}$ i.a./ha): captan $(1250,0)$, fenarimol $(80,0)$, trifenil hidróxido de estanho $(166,7)$, prochloraz $(450,0)$, chlorothalonil $(1500,0)$, iprodione $(750,0)$, azoxystrobin $(150,0)$, thiabendazole $(360,0)$, procimidone $(750,0), \mathrm{zi}$ ram $(1500,0)$ e tebuconazole $(200,0)$. Parcelas sem aplicação de fungicida constituíram a testemunha. As pulverizações foram realizadas com pulverizador costal manual, com o jato dirigido para as panículas. O intervalo das aplicações dos fungicidas foi de 4 dias, a partir da antese. Aos 7 dias após o término das pulverizações, a porcentagem de panículas doentes variou 5,4 para o fungicida tebuconazole e $100,0 \%$ para a maioria dos
\end{abstract}

demais tratamentos. Para a porcentagem de flores doentes por panícula, os valores variaram de $0,3 \%$ a $65,0 \%$ para o fungicida tebuconazole e testemunha, respectivamente. Não ocorreu diferença significativa entre as porcentagens de flores doentes por panículas nos tratamentos tebuconazole $(0,3 \%)$, chlorothalonil $(2,5 \%)$ e prochloraz $(3,0 \%)$, sendo esses os mais eficientes no controle da ergot. Adicionalmente, o fungicida mais eficiente no controle da antracnose foliar (Colletotrichum graminicola) foi o prochloraz, ao passo que para a ferrugem (Puccinia purpurea) e a cercosporiose (Cercospora fusimaculans), o maior controle foi obtido com o fungicida tebuconazole. Não houve aumento da germinação das sementes em nenhum dos tratamentos; entretanto, o aumento do peso de mil sementes foi significativo para os tratamentos com os fungicidas prochloraz, chlorotalonil e tebuconazole.

TERMOS PARA INDEXAÇÃO: Sorghum bicolor, fungos, doenças, produção de sementes.

\section{CHEMICAL CONTROL OF "ERGOT"(Claviceps africana Frederickson, Mantle \& de Milliano) OR SUGARY DISEASE AND THE MAIN FOLIAR DISEASES OF SORGHUM (Sorghum bicolor (L.) Moench)}

\begin{abstract}
The efficiency of fungicides in the control of sugar disease of sorghum (Sorghum bicolor (L.) Moench.) was evaluated in a seed production field of the sorghum hybrid BR 304. The following fungicides and doses ( $\mathrm{g}$ a.i./ha) were evaluated : captan (1250.0), fenarimol (80.0), fentin hydroxide (166.7), prochloraz (450.0), chlorothalonil (1500.0), iprodione (750.0), azoxystrobin (150.0), thiabendazole (360.0), procimidone (750.0), ziram (1500.0) and tebuconazole (200.0). A control plot without fungicide application was included in this assay. Applications were performed with a back sprayer, covering the whole panicle, and using 4 days intervals between applications at the anthesis. Seven days after the end of the
\end{abstract}

applications, the percentage of diseased panicles varied of 5.4 for the fungicide tebuconazole and 100 for the most of the other treatments. For the percentage of diseased flowers for panicle, the values varied from 0.3 to 65.0 for the fungicide tebuconazole and check, respectively. No significant difference among the percentages of diseased flowers for panicles in the treatments tebuconazole (0.3), chlorothalonil (2.5) and prochloraz (3.0), being those the most efficient fungicides in the control of the "ergot". However, the fungicide prochloraz was the most efficient in the control of foliar disease casel by Colletotrichum graminicola, while for the fungi Puccinia purpurea, and Cercospora fusimaculans the best control were

1. Engenheiro Agrônomo, Doutor em Agronomia, Pesquisador da Embrapa Milho e Sorgo, Laboratório de Patologia de Sementes e Grãos, Caixa Postal 151, 35701-970, Sete Lagoas, MG. nicesio@cnpms.embrapa.br 
obtained with the tebuconazole. There was not an increase of the germination in none of the treatments, however the increase in the weight of a thousand seeds was significant for the treatments with prochloraz, chlorothalonil and tebuconazole.

INDEX TERMS: Sorghum bicolor, fungi, diseases, seed production.

Entre as inúmeras doenças a que está sujeita a cultura do sorgo, a "ergot", ou doença-açucarada, causada por Sphacelia sorghi (Claviceps africana), tem sido relatada em diversos países da África, Ásia, América do Sul e América do Norte. No Brasil, foi constatada pela primeira vez em 1995, sendo o agente etiológico classificado por Ferreira \& Casela (1995) em suas formas anamórfica e teleomórfica como $S$ phacelia sorghi e Claviceps sorghi, respectivamente, sendo posteriormente sua forma teleomórfica reclassificada como Claviceps africana (Reis et al., 1996). Esse fungo infecta os ovários das flores de sorgo, sendo considerado um patógeno exclusivo dos órgãos reprodutivos.

A doença-açucarada do sorgo tem sido considerada uma grande ameaça para a produção de sementes híbridas no Brasil (Pinto et al., 1999). A severidade dessa doença é maior quando a umidade relativa do ar está acima de $80 \%$ e a temperatura $19 \pm 1^{\circ} \mathrm{C}$ durante o período da antese (Bandyopadhyay, 1992). Ademais, o período de suscetibilidade dos ovários da flor de sorgo à Sphacelia sorghi reduz a zero no quinto dia após a polinização (Puranik et al., 1973).

A doença-açucarada afeta principalmente a produção de sementes híbridas, uma vez que linhagens com macho-esterilidade citoplasmática usadas como progenitores femininos são altamente suscetíveis ao patógeno. Nas situações em que a fertilização é retardada devido à ausência de pólen viável causada pela não-coincidência de florescimento entre a linhagem macho-estéril e a linhagem restauradora, a produção de sementes híbridas pode ser drasticamente afetada. Além disso, as condições ambientais favoráveis ao desenvolvimento da doença são prejudiciais à fertilização, tornando as espiguetas mais vulneráveis ao ataque da doença (Casela et al., 1997)

Poucos trabalhos sobre o controle químico de C. africana estão disponíveis na literatura. Há relatos de que a atividade residual dos fungicidas benomyl, bitertanol, carbendazim + flusilazole, procymidone, propiconazole, tebuconazole e triadimenol, utilizados em aplicação única, não controlou Claviceps africana durante o período de florescimento da panícula (McLaren, 1994). Por outro lado, os fungicidas mancozeb, captafol, carbendazim + tridemorfo, carbendazim
+ thiram e tiofanato metílico foram eficientes na redução da doença e aumentaram significativamente a produção de grãos (Anahosur, 1979; Lakshmanan \& Mohan, 1988). Por meio de estudos "in-vitro", verificou-se que os fungicidas benomyl, captan, ziram, thiram, carboxin e oxicloreto de cobre + zinco inibiram o crescimento e a esporulação de Sphacelia sorghi (Khadke et al., 1979). No Brasil, Pinto et al. (1997) relataram a eficiência dos fungicidas tebuconazole, propiconazole e triadimenol no controle desse patógeno. De acordo com Pinto (1999), os fungicidas propiconazole, propiconazole + difenoconazole e tebuconazole foram eficientes no controle da doençaaçucarada em sorgo, tendo sido observado em relação aos produtos tebuconazole e propiconazole + difenoconazole também o controle das doenças foliares causadas por Puccinia purpurea (ferrugem), Colletotrichum graminicola (antracnose), Exserohilum turcicum (helmintosporiose) e Cercospora fusimaculans (cercosporiose), as quais estão relacionadas entre as principais doenças foliares da cultura do sorgo no Brasil (Casela et al., 1997).

Conduziu-se este trabalho com os objetivos de avaliar a eficiência de fungicidas no controle da doençaaçucarada do sorgo e também as suas ações sobre as doenças foliares do sorgo.

Estabeleceu-se em fevereiro de 1998 um campo de produção de sementes do híbrido de sorgo BR 304, composto pelas linhagens BR 001A (macho-estéril) e BR 012 (restauradora). A linhagem BR 001A, utilizada para o cruzamento, foi plantada dez dias após a BR 012, na proporção de 4:2. Para garantir a fonte primária artificial de inóculo, foi plantada lateralmente a cada parcela a linhagem BR 001A, sendo inoculada na antese com uma suspensão de $10^{6}$ conídios/ml, funcionando como linhas disseminadoras do fungo. Assim, o campo de produção de sementes ficou com as parcelas compostas de quatro fileiras de BR 001A, duas fileiras de BR 012 e duas fileiras de BR 001A disseminadoras de inóculo.

$\mathrm{O}$ delineamento experimental foi o de blocos ao acaso, com 12 tratamentos em 3 repetições, com parcelas compostas de quatro fileiras (BR 001A) com $7 \mathrm{~m}$ de comprimento. Foram utilizados os seguintes fungicidas com doses expressas em $\mathrm{g}$ i.a./ha : captan $(1250,0)$, fenarimol $(80,0)$, trifenil hidróxido 
de estanho (166,7), prochloraz $(450,0)$, chlorothalonil $(1500,0)$, iprodione $(750,0)$, azoxystrobin $(150,0)$, thiabendazole $(360,0)$, procimidone $(750,0)$, ziram $(1500,0)$ e tebuconazole $(200,0)$. Parcelas sem aplicação de fungicida constituíram a testemunha.

As aplicações dos fungicidas foram realizadas empregando-se pulverizador costal manual, com o jato dirigido para as panículas. $\mathrm{O}$ intervalo programado para 3 aplicações dos fungicidas foi de 4 dias, a partir da antese. Em razão da ocorrência de chuvas, no dia seguinte repetiam-se as aplicações, pois a falta de transpiração das flores faz com que os fungicidas sistêmicos atuem com ação de contato, devido ao impedimento da entrada desses fungicidas nos espaços livres dos tecidos (Crowdy, 1977), visto que as flores não possuem estômatos.

Aos sete dias após a última aplicação dos fungicidas, determinaram-se, nas plantas das duas fileiras centrais das parcelas, a porcentagem de panículas com sintomas da doença-açucarada, bem como a porcentagem de flores doentes por panícula. Para a análise de variância, os dados originais foram transformados por arc sen $\sqrt{\text { porcentagem/100 }}$. A germinação das sementes oriundas das parcelas experimentais e o peso de mil sementes foram determinados de acordo com as Regras para Análise de Sementes (Brasil, 1992). A avaliação das doenças foliares foi realizada quando as sementes atingiram o estádio da maturidade fisiológica, empregando-se uma escala de notas de 0 a 5 ( $0=$ ausência de lesões; $1=$ lesões esparsas; $2=$ lesões em $50 \%$ das folhas e com $25 \%$ de severidade; $3=$ lesões em $75 \%$ das folhas e com $50 \%$ de severidade; $4=$ lesões em $100 \%$ das folhas e com $75 \%$ de severidade; e $5=$ lesões em $100 \%$ das folhas e com seca total das plantas) (Pinto \& Fernandes, 1995).

Salienta-se que no período em que o sorgo atingiu o estádio de polinização e fertilização, e se procederam às pulverizações com os fungicidas (abril de 1998), as médias da temperatura máxima, da temperatura mínima, da umidade relativa do ar e da precipitação pluviométrica foram de $29,8^{\circ} \mathrm{C}, 18,1^{\circ} \mathrm{C}, 72,0 \%$ e $37,5 \mathrm{~mm}$, respectivamente.

Os resultados obtidos no controle da doençaaçucarada estão na Tabela 1, na qual se observa que para a porcentagem de panículas doentes, apenas o fungi- cida tebuconazole foi eficiente, e os demais tiveram o comportamento semelhante ao da testemunha. Esses resultados estão de conformidade com aqueles apresentados por Anahosur (1979), Lakshmanan \& Mohan (1988) e Pinto (1999).

Com relação à porcentagem de flores doentes por panícula, os fungicidas tebuconazole, prochloraz e chlorotalonil foram os que apresentaram o melhor controle. A redução da porcentagem de flores doentes por panícula mediante a aplicação de tebuconazole está de acordo com os resultados apresentados por Pinto (1999).

Nenhum tratamento produziu aumento significativo na germinação das sementes. Observou-se, entretanto, que o aumento do peso de mil sementes foi significativo para os tratamentos com os fungicidas prochloraz, chlorotalonil e tebuconazole. Ficou evidenciado que o fungicida trifenil hidróxido de estanho apresentou fitoxicidade à cultura do sorgo, confirmando os resultados obtidos por Pinto et al. (1997).

Os resultados da incidência e da severidade das doenças foliares na linhagem macho-estéril (BR 001A) estão apresentados na Tabela 2, na qual se observa que: 1) o fungicida mais eficiente no controle da antracnose foliar (Colletotrichum graminicola) foi o prochloraz, 2) para o controle da ferrugem (Puccinia purpurea), foram mais eficientes os fungicidas tebuconazole e azoxystrobin, e 3) para a cercosporiose (Cercospora fusimaculans), o melhor controle foi obtido com os fungicidas tebuconazole, fenarimol, chlorotalonil e thiabendazole. Resultados semelhantes aos obtidos neste trabalho com o fungicida tebuconazole foram reportados por Pinto (1999). Do exposto, pode-se inferir: 1- Os fungicidas tebuconazole, prochloraz e chlorotalonil são eficientes no controle da ergot ou doença-açucarada do sorgo; 2O fungicida prochloraz é eficiente no controle da antracnose (Colletotrichum graminicola) do sorgo; 3- Os fungicidas tebuconazole e azoxystrobin são eficientes no controle da ferrugem (Puccinia purpurea) do sorgo; e 4- Os fungicidas tebuconazole, fenarimol, chlorotalonil e thiabendazole são eficientes no controle da cercosporiose (Cercospora fusimaculans) do sorgo. 
TABELA 1 - Porcentagem de panículas doentes e de flores doentes por panículas, oriundas de parcelas tratadas com fungicidas, em campo de produção de sementes de sorgo híbrido da cultivar BR 304. Embrapa Milho e Sorgo, Sete Lagoas, MG, 1998.

\begin{tabular}{|c|c|c|c|c|c|}
\hline Tratamento & $\begin{array}{c}\text { Dose } \\
{\text { (g i.a. })^{1}}^{1}\end{array}$ & $\begin{array}{c}\text { PD } \\
(\%)^{2}\end{array}$ & $\begin{array}{c}\text { FD } \\
(\%)^{3}\end{array}$ & $\begin{array}{l}\text { TPG } \\
(\%)^{4}\end{array}$ & $\begin{array}{c}\text { PMS } \\
(\mathrm{g})^{5}\end{array}$ \\
\hline Captan & 1250,0 & $100,0 \mathrm{a}$ & $33,3 b c^{6}$ & $91,7 \mathrm{a}$ & $26,0 \mathrm{~cd}$ \\
\hline Fenarimol & 80,0 & $95,7 \mathrm{a}$ & $12,7 \mathrm{~d}$ & $91,3 \mathrm{a}$ & $33,0 \mathrm{abc}$ \\
\hline Trifenil hidróxido de estanho & 166,7 & $*$ & $*$ & $*$ & $*$ \\
\hline Prochloraz & 450,0 & $96,0 \mathrm{a}$ & $3,0 \mathrm{e}$ & $90,7 \mathrm{a}$ & $36,3 \mathrm{a}$ \\
\hline Chlorothalonil & 1500,0 & $98,8 \mathrm{a}$ & $2,5 \mathrm{e}$ & $91,3 \mathrm{a}$ & $36,1 \mathrm{a}$ \\
\hline Iprodione & 750,0 & $100,0 \mathrm{a}$ & $20,0 \mathrm{~cd}$ & 89,7 a & $28,8 \mathrm{bcd}$ \\
\hline Azoxystrobin & 150,0 & $100,0 \mathrm{a}$ & $25,0 \mathrm{~cd}$ & $89,7 \mathrm{a}$ & $27,3 \mathrm{~cd}$ \\
\hline Thiabendazole & 360,0 & $100,0 \mathrm{a}$ & $43,3 \mathrm{~b}$ & 90,0 a & $26,4 \mathrm{~cd}$ \\
\hline Procimidone & 750,0 & $100,0 \mathrm{a}$ & $40,0 \mathrm{~b}$ & $92,3 \mathrm{a}$ & $26,8 \mathrm{~cd}$ \\
\hline Ziram & 1500,0 & $100,0 \mathrm{a}$ & $25,0 \mathrm{~cd}$ & 90,3 a & $27,9 \mathrm{~cd}$ \\
\hline Tebuconazole & 200,0 & $5,4 \mathrm{~b}$ & $0,3 \mathrm{e}$ & $91,7 \mathrm{a}$ & $38,9 \mathrm{a}$ \\
\hline Testemunha & ---- & $100,0 \mathrm{a}$ & $65,0 \mathrm{a}$ & $88,7 \mathrm{a}$ & $22,8 \mathrm{~d}$ \\
\hline C.V. (\%) & & 6,0 & 12,2 & 2,1 & 8,4 \\
\hline
\end{tabular}

${ }^{1}$ Dose expressa em gramas do ingrediente ativo / ha

${ }^{2}$ Panículas doentes : dados originais transformados por arc sen $\sqrt{\text { porcentagem / } 100}$

${ }^{3}$ Flores doentes / panícula : dados originais transformados por arc sen $\sqrt{\text { porcentagem /100 }}$

${ }^{4}$ Teste padrão de germinação

${ }^{5}$ Peso de mil sementes

${ }^{6}$ Numa coluna, as médias seguidas pelas mesmas letras não diferem entre si (Tukey $5 \%$ )

(*) Não avaliado devido à ocorrência de efeito fitotóxico 
TABELA 2 - Eficiência de fungicidas no controle de doenças foliares na linhagem de sorgo BR 001A, quando aplicados para o controle da "ergot" (Claviceps africana) ou doença-açucarada. Embrapa Milho e Sorgo, Sete Lagoas, MG, 1998.

\begin{tabular}{lcccc}
\hline \multicolumn{1}{c}{ Tratamento } & Dose $(\mathbf{g} \text { i.a. })^{\mathbf{1}}$ & Antracnose & Ferrugem & Cercospora \\
\hline Captan & 1250,0 & $3,5^{2}$ & 0,7 & 0,3 \\
Fenarimol & 80,0 & 3,5 & 1,7 & 0,0 \\
Trifenil hidróxido de estanho & 166,7 & $*$ & $*$ & $*$ \\
Prochloraz & 450,0 & 1,0 & 3,0 & 0,0 \\
Chlorothalonil & 1500,0 & 2,0 & 1,0 & 0,8 \\
Iprodione & 750,0 & 3,0 & 1,0 & 1,2 \\
Azoxystrobin & 150,0 & 2,0 & 0,0 & 0,3 \\
Thiabendazole & 360,0 & 2,0 & 3,0 & 0,0 \\
Procimidone & 750,0 & 3,8 & 1,0 & 1,0 \\
Ziram & 1500,0 & 3,0 & 1,0 & 1,2 \\
Tebuconazole & 200,0 & 2,0 & 0,0 & 0,0 \\
\hline Testemunha & ---- & 4,5 & 3,0 & 2,0 \\
\hline
\end{tabular}

${ }^{1}$ Dose expressa em gramas do ingrediente ativo/ha

${ }^{2}$ Média de três repetições por tratamento. Escala de notas : 0 a 5 de acordo com Pinto \& Fernandes (1995).

(*) Não avaliado devido à ocorrência de efeito fitotóxico

\section{AGRADECIMENTOS}

A Osni Alves da Silva e José Moreira Campos (Assistentes de Pesquisa), e a Gilberto Ribeiro Rodrigues e Almir Roberto da Silva (Operários Rurais), pela valiosa contribuição na execução deste trabalho.

\section{REFERÊNCIAS BIBLIOGRÁFICAS}

ANAHOSUR, K. H. Chemical control of ergot of sorghum. Indian Phytopathology, New Delhi, v. 32, n. 3, p. 487-489, Sept. 1979.

BANDYOPADHYAY, R. Sorghum Ergot. In: MILLIANO, W. A. J. de; FREDERIKSEN, R. A.; BENGSTON, G. D. (Eds.). Sorghum and millets diseases: a second world review. Patancheru: ICRISAT, 1992. p. 235-244.

BRASIL. Ministério da Agricultura e Reforma Agrária. Regras para Análise de Sementes. Brasília, DF, 1992. $365 \mathrm{p}$.
CASELA, C. R.; PINTO, N. F. J. A.; OLIVEIRA, E.; FERREIRA, A. S. Sorgo: Sorghum bicolor (L.) Moench controle de doenças. In: VALE, F. X. R. do; ZAMBOLIM, L. (Eds.). Controle de doenças de plantas: grandes culturas. Viçosa: UFV/Departamento de Fitopatologia, 1997. cap. 22, p. 1025-1063.

CROWDY, S. H. Translocation. In: MARSH, R. W. (Ed.). Systemic fungicides. 2. ed. London: Longman, 1977. cap. 5, p. 92-114.

FERREIRA, A. S.; CASELA, C. R. Ocorrência de Claviceps sorghi agente causal da doença ergot no Brasil. Fitopatologia Brasileira, Brasília, v. 20, p. 302, ago. 1995. Suplemento.

KHADKE, V. D.; MORE, B. B.; KONDE, B. K. In vitro evaluation of some fungicides and antibiotics for the control of Sphacelia sorghi an incitant of sugary disease of sorghum. Pesticides, Bombay, v. 12, n. 7, p. 59-60, July 1979. 
LAKSHMANAN, P.; MOHAN, S. Studies on the effect of various fungicides on Sphacelia sorghi. Pesticides, Bombay, v. 22, n. 9, p. 27, Sept. 1988.

McLAREN, N. W. Efficacy of systemic fungicides and timing of preventative sprays in the control of sugary disease of grain sorghum (Sorghum bicolor). South African Journal of Plant and Soil, Pretoria, v. 11, n. 1, p. 30-33, May 1994.

PINTO, N. F. J. A. Avaliação de fungicidas no controle de Sphacelia sorghi (Claviceps africana) agente etiológico da "ergot" ou doença açucarada do sorgo. Summa Phytopathologica, Jaguariuna, v. 25, n. 1, p. 4-8, jan./mar. 1999.

PINTO, N. F. J. A.; FERNANDES, F. T. Avaliação de fungicidas no controle da mancha foliar do milho causada por Phyllosticta sp. (Phaeosphaeria maydis). Fitopatologia Brasileira, Brasília, v. 20, p. 333, ago. 1995. Suplemento.
PINTO, N. F. J. A.; FERREIRA, A. S.; CASELA, C. R. Chemical control of sugary disease of sorghum (Claviceps africana). In: THE GLOBAL CONFERENCE ON ERGOT OF SORGHUM, 1997, Sete Lagoas. Proceedings... Sete Lagoas: EMBRAPA/INTSORMIL, 1999. p. 158-160. (Publication, n. 99-1).

PINTO, N. F. J. A.; FERREIRA, A. S.; CASELA, C. R. Ergot (Claviceps africana) ou doença açucarada do sorgo. Sete Lagoas: EMBRAPA-CNPMS, 1997. 24 p. (EMBRAPA-CNPMS. Circular Técnica, 23).

PURANIK, S. B.; PADAGANUR, G. M.; HIREMATH, R. V. Susceptibility period of sorghum ovaries to Sphacelia sorghi. Indian Phytopathology, New Delhi, v. 26, n. 3, p. 586-587, Sept. 1973.

REIS, E. M.; MANTLE, P. G.; HASSAN, H. A. G. First report in the Americas of sorghum ergot disease, caused by a pathogen diagnosed as Claviceps africana. Plant Disease, Beltsville, v. 80, n. 4, p. 463, Apr. 1996. 